\title{
Predicción de rugosidad en maquinado de compuestos con base de Peek usando metodología de superficie de respuesta
}

\section{Predictive Models for Different Roughness Parameters During Machining Process of Peek Composites Using Response Surface Methodology}

\author{
Mata-Cabrera Francisco \\ Escuela de Ingeniería Minera e Industrial de Almadén \\ Ciudad Real, España \\ Correo: Francisco.MCabrera@uclm.es \\ Hanafi Issam \\ Facultad de Ciencias de Tetuán, Marruecos \\ Correo:hanafi.issam@yahoo.fr \\ Khamlichi Abdellatif \\ Facultad de Ciencias de Tetuán, Marruecos \\ Correo:Khamlichi7@yahoo.es
}

\author{
Jabbouri Abdallah \\ Facultad de Ciencias y Técnicas de Tánger, Marruecos \\ Correo:a.jabbouri@gmail.com \\ Bezzazi Mohamed \\ Facultad de Ciencias y Técnicas de Tánger, Marruecos \\ Correo:bezzazi@hotmail.com
}

Información del artículo: recibido: marzo de 2010, reevaluado: abril de 2010 y mayo de 2012, aceptado: febrero de 2013

\section{Resumen}

La polieteretercetona (PEEK) pertenece a un grupo de polímeros termoplásticos de alto rendimiento que se utiliza ampliamente en componentes estructurales. Para mejorar las propiedades tribológicas y mecánicas, se añaden fibras cortas a los materiales termoplásticos. Por lo tanto, es necesario investigar el mecanizado de estos compuestos debido a las altas propiedades específicas y a sus usos potenciales. Este trabajo presenta la utilización de la metodología de superficie de respuesta (MSR) para estudiar los aspectos de la maquinabilidad del PEEK no reforzado (PEEK), del PEEK reforzado con $30 \%$ de fibras de carbono (PEEK CF30) y del PEEK reforzado con $30 \%$ de fibras de vidrio (PEEK GF30) con una herramienta de carburo cementado (K10). Este estudio establece las relaciones entre las condiciones de corte (velocidad de corte $V_{c}$ y velocidad de avance $\left.V_{a}\right)$ y la rugosidad $\left(R_{t}, R_{a}, R_{q^{\prime}} R_{p}\right)$, desarrollando modelos matemáticos de segundo orden. Se ha utilizado el diseño factorial para establecer el plan de experimentos y el análisis de varianza para comprobar la suficiencia de los modelos. Los modelos de predicción han mostrado validez dentro del rango de parámetros de corte utilizados. En lo que respecta a la influencia de las condiciones de corte, se ha demostrado que la variación de la velocidad de avance, dentro de los valores seleccionados, afecta más a los resultados que la variación de la

\section{Descriptores:}

- polieteretercetonas

- polímeros termoplásticos

- metodología de superficie de respuesta

- rugosidad

- diseño factorial

- análisis de varianza 
velocidad de corte. Los resultados de los ensayos han mostrado que los materiales reforzados presentan una peor maquinabilidad en comparación con el termoplástico sin refuerzo. En este caso, el PEEK GF30 es el material que evidencia una peor maquinabilidad.

\begin{abstract}
Polyetheretherketone (PEEK) composite belongs to a group of high performance thermoplastic polymers and is widely used in structural components. To improve the mechanical and tribological properties, short fibers are added as reinforcement to the material. Due to its functional properties and potential applications, it's important to investigate the machinability of non-reinforced PEEK (PEEK), PEEK reinforced with $30 \%$ of carbon fibers (PEEK CF30), and reinforced PEEK with 30\% glass fibers (PEEK GF30) to determine the optimal conditions for the manufacture of the parts. The present study establishes the relationship between the cutting conditions (cutting speed and feed rate) and the roughness $\left(R_{a}, R_{t}, R_{q}, R_{p}\right)$, by developing second order mathematical models. The experiments were planned as per full factorial design of experiments and an analysis of variance has been performed to check the adequacy of the models. These state the adequacy of the derived models to obtain predictions for roughness parameters within ranges of parameters that have been investigated during the experiments. The experimental results show that the most influence of the cutting parameters is the feed rate, furthermore, proved that glass fiber reinforcements produce a worse machinability.
\end{abstract}

Keywords:

- polyetheretherketone

- thermoplastic polymers

- response surface methodology

- roughness

- factorial design

- analysis of variance

\section{Introducción}

La polieteretercetona (PEEK) pertenece a un grupo de polímeros termoplásticos de alto rendimiento. Los compuestos de PEEK se han utilizado ampliamente en automóviles, aeronáutica, biomecánica, robots y máquinas debido a sus altas propiedades específicas respecto a los materiales metálicos convencionales. También encuentran aplicaciones en las industrias naval y militar, en la construcción e ingeniería civil o en la industria química, entre otras.

El material PEEK es el polímero que exhibe propiedades como elevadas resistencias mecánicas, resistencia al desgaste y a la abrasión, resistencia a los agentes químicos y puede trabajar a temperaturas elevadas (Davim et al., 2003; Davim y Mata, 2006). Debido a sus propiedades, este material polimérico se aplica con excepcionales prestaciones en la ingeniería, por ejemplo en la industria aeroespacial, donde el aluminio se ha sustituido por el PEEK debido su mejor comportamiento a temperaturas altas (Davim y Mata, 2007; Davim y Mata, 2008).

Para mejorar las propiedades tribológicas y mecánicas, como la rigidez o el límite elástico, se añaden fibras cortas a los termoplásticos (Davim y Mata, 2005; Davim y Reis, 2004). Las fibras de carbono y de vidrio son los refuerzos más comunes en termoplásticos debido a su alto módulo de elasticidad. Gracias a las altas resistencia y rigidez, los refuerzos de fibra encuentran muchas aplicaciones en componentes resistentes o estructurales. El PEEK reforzado con fibras de vidrio (PEEK GF30) y el PEEK reforzado con fibras de carbono (PEEK CF30) son los mejores materiales para aplicaciones que requieren alta resistencia y rigidez, especialmente a temperaturas por encima de los $150^{\circ} \mathrm{C}$ (Harsha y Tewari, 2003; Sinmazcelik y Yilmaz, 2007; Burris y Sawyer, 2007).

Como consecuencia de las propiedades y aplicaciones potenciales, existe una gran necesidad de conocer los procesos de fabricación, en particular los procesos de mecanizado de estos materiales compuestos (Cogswell, 1992; Kukureka et al., 1999; Miravete et al., 2000; Mallick, 2001; Park et al., 2006; Lee et al., 2006; Kim et al., 2006; Verrey et al., 2006).

En la actualidad, crece progresivamente la demanda de componentes fabricados con materiales plásticos reforzados con fibras, debido a su precisión dimensional y a su acabado superficial (Palanikumar et al., 2004; Palanikumar, 2006). El estudio de las superficies constituye una técnica de caracterización de los materiales muy útil en la práctica.

Al mecanizar la polieteretercetona reforzada se evidencia un comportamiento extremadamente abrasivo. Las propiedades y características del material de traba- 
jo se evalúan en términos de "maquinabilidad", que indica la facilidad relativa con la cual un material se puede maquinar usando las herramientas y los parámetros apropiados. Los criterios más importantes para evaluar la maquinabilidad son la fuerza de corte, la presión específica de corte, la vida de la herramienta, el desgaste de la herramienta y la aspereza superficial o rugosidad. La rugosidad superficial es un parámetro que tiene gran influencia en el comportamiento y la funcionalidad de los componentes mecánicos y en los costos de producción (Abouelatta y Mádl, 2001; Palanikumar et al., 2006), constituyendo una importante variable de control de la calidad. La rugosidad es crítica en los contactos mecánicos, además de otros campos, como la circulación de fluidos y las aplicaciones semiconductoras.

En la mayoría de las aplicaciones se requiere alta calidad en las superficies mecanizadas, incluyendo exactitud dimensional e integridad superficial. Por esta razón, se han llevado a cabo diversas investigaciones con el objetivo de optimizar los parámetros de corte, para obtener una determinada rugosidad (Eriksen, 1999; Abouelatta y Mádl, 2001).

Al hablar de parámetros funcionales de corte nos referimos a la selección de la velocidad de corte, la velocidad de avance y la profundidad de corte. Se han realizado diferentes estudios sobre los parámetros de corte adecuados en la operación de torneado (Yang y Tarng., 1998; El-Sonbaty et al., 2004; Varatharajan et al., 2006), de los que se puede concluir que no existe una referencia universal; antes bien, cada material, según el tipo y porcentaje de fibras de refuerzo, presenta unos valores de velocidad de corte y velocidad de avance más apropiados para conseguir un acabado superficial óptimo y minimizar el desgaste de la herramienta.

Bernardos y Vosniakos presentaron diferentes metodologías que emplearon para predecir la rugosidad superficial (diseño de experimentos, inteligencia artificial, etcétera) y consideraron la influencia de los parámetros de mecanizado, de las propiedades de las herramientas de corte, de las propiedades de la pieza y del fenómeno de corte sobre la rugosidad (Bernardos y Vosniakos, 2003).

Davim y Reis presentaron un estudio sobre la influencia de los parámetros de corte (velocidad de corte y avance) en la rugosidad y en la precisión dimensional torneando tubos de plásticos reforzados con fibras (FRP). Utilizaron matrices ortogonales y análisis de varianza (ANOVA) para investigar las características de corte de los FRP usando insertos de carburos cementados (K15) (Davim y Reis, 2004).
El método de investigación experimental tradicional implica la variación de un parámetro mientras se mantienen otros parámetros en niveles fijos. El método es laborioso, requiere un gran número de experimentos y no incluye efectos interactivos entre los factores controlables. El método de Taguchi, sin embargo, permite enriquecer los métodos de planes de experiencias y tiene por objeto simplificar el protocolo experimental con el fin de poner de relieve los efectos de los factores sobre la respuesta.

La metodología de superficie de respuesta es un conjunto de técnicas matemáticas y estadísticas utilizadas para modelar y analizar problemas en los que una variable de interés es influenciada por otras. El objetivo es optimizar la variable de interés.

Este estudio establece una correlación entre los parámetros de corte (velocidad de corte $V_{c}$ y velocidad de avance $\left.V_{a}\right)$ y la rugosidad $\left(R_{t}, R_{q}, R_{a}, R_{p}\right)$, desarrollando modelos matemáticos de segundo orden. Se ha utilizado diseño factorial para establecer el plan de experimentos y análisis de varianza para comprobar la suficiencia de los modelos.

\section{Trabajo experimental}

\section{Materiales ensayados}

Para realizar este trabajo de investigación sobre el mecanizado de materiales compuestos, se han utilizado materiales compuestos de matriz polimérica termoplástica, en presentación natural (sin refuerzo) o reforzados con porcentajes fijos de fibras de vidrio o de carbono. En la actualidad, las investigaciones básicas realizadas en tecnología de materiales han permitido procesar materiales termoplásticos que aúnan propiedades y desempeños excelentes en un buen número de aplicaciones y constituyen uno de los mayores focos de atención de la investigación aplicada, especialmente en lo referente al mecanizado por arranque de material.

En concreto, los materiales compuestos de matriz polimérica utilizados en los ensayos son los siguientes:

a) Polieteretercetona (PEEK)

b) Polieteretercetona reforzada con $30 \%$ de fibras de vidrio (PEEK GF 30)

c) Polieteretercetona reforzada con $30 \%$ de fibras de carbono (PEEK CF 30)

Las propiedades más significativas de estos materiales quedan reflejadas en la tabla 1. 
Tabla 1. Propiedades de los materiales ensayados (ERTA®)

\begin{tabular}{|c|c|c|c|c|}
\hline Propiedad & PEEK & PEEK GF30 & PEEK CF30 & Unidad \\
\hline Módulo de elasticidad (E) & 4400 & 6300 & 7700 & $\mathrm{MPa}$ \\
\hline Dureza Rockwell & M105 & M99 & M102 & - \\
\hline Resistencia al impacto & Sin fractura & 35 & 35 & $\mathrm{~kJ} / \mathrm{m}^{2}$ \\
\hline Temperatura de fusión & 340 & 340 & 340 & ${ }^{\circ} \mathrm{C}$ \\
\hline Densidad & 1.31 & 1.51 & 1.41 & $\mathrm{~g} / \mathrm{cm}^{3}$ \\
\hline Coeficiente de expansión térmica $\left(<150^{\circ} \mathrm{C}\right)$ & $50 \times 10^{-6}$ & $30 \times 10^{-6}$ & $25 \times 10^{-6}$ & $\mathrm{~m} / \mathrm{m} / \mathrm{K}$ \\
\hline Coeficiente de expansión térmica $\left(>150^{\circ} \mathrm{C}\right)$ & $110 \times 10^{-6}$ & $65 \times 10^{-6}$ & $55 \times 10^{-6}$ & $\mathrm{~m} / \mathrm{m} / \mathrm{K}$ \\
\hline
\end{tabular}

Preparación de las probetas

Dado que el objetivo de este trabajo de investigación es estudiar el proceso de torneado de piezas pequeñas de materiales compuestos de matriz polimérica termoplástica, se diseñaron probetas de $100 \mathrm{~mm}$ de longitud. El material base se adquirió en forma de redondos extruidos de $50 \mathrm{~mm}$ de diámetro, de modo que las dimensiones de las probetas son longitud: $100 \mathrm{~mm}$ y diámetro inicial: $50 \mathrm{~mm}$.

\section{Máquinas y herramientas}

\section{Torno CNC}

Para la realización de los ensayos experimentales de torneado se ha utilizado un torno CNC "Kingsbury ${ }^{\circledR}$ MHP 50" de $18 \mathrm{~kW}$ de potencia y velocidad máxima de $4500 \mathrm{rpm}$. El torno CNC posee un controlador Fanuc, una boca de apriete hidráulico, capaz de fijar piezas de hasta $200 \mathrm{~mm}$ de diámetro, una torreta para almacenamiento de las herramientas, con capacidad para 12 herramientas de corte. En nuestro estudio se utilizó la herramienta K10 (DCMW 11T304H13A) y un contrapunto con diámetro de $70 \mathrm{~mm}$ activado también por accionamiento hidráulico.

\section{Rugosidad superficial}

La caracterización de las superficies torneadas se efectuó a través de la técnica más común de análisis de microgeometría de superficies, denominada rugosidad. Las superficies mecanizadas se caracterizaron utilizando los parámetros de amplitud siguientes: $R_{t}, R_{q}, R_{a}$ y $R_{p}$. Para medir los valores de los parámetros de rugosidad, se utilizó un rugosímetro Hommeltester - T 1000, según se puede observar en la figura 2.

La rugosidad media aritmética $\left(R_{a}\right)$ indica el valor medio de la rugosidad en una zona de la muestra. Es el parámetro de caracterización de rugosidad que más se utiliza en la mayoría de los procesos de fabricación y se determina mediante la expresión:

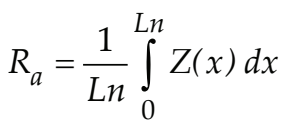

donde, $L n$ es la longitud de evaluación y $Z$ es la distancia entre dos puntos del perfil.

La amplitud máxima del perfil de rugosidad $\left(R_{t}\right)$ Corresponde a la distancia entre el pico más alto y el valle más profundo obtenidos en la longitud de medida $(L n)$, que es independiente de los valores parciales de rugosidad. Se trata de un parámetro que, a diferencia del anterior, tiene un claro significado físico. La altura máxima de picos $\left(R_{p}\right)$ es la distancia del punto más alto del perfil a la línea media. Finalmente, la desviación media cuadrática del perfil $\left(R_{q}\right)$ es el valor cuadrático medio de las desviaciones.

\section{Diseño de experimentos}

El proceso de torneado se desarrolló sin lubricante y considerando las doce combinaciones de los tres valores de la velocidad de corte y de los cuatro valores de la velocidad de avance, de acuerdo con la tabla 2 . Se utilizó una profundidad de pasada constante de $2 \mathrm{~mm}$. Para los experimentos considerados en este estudio, se empleó un plan experimental basado en el método de Taguchi (1990), lo que permitió reducir el número de ensayos y el costo asociado a los mismos. En la determinación de la primera aproximación del grado de influencia de los parámetros, utilizamos el diseño factorial completo, donde los factores identificados en las pruebas adoptan sus límites superior e inferior. En nuestro caso, sólo se seleccionaron dos factores, de manera que las posibles combinaciones de los niveles coresponden a un total de 12 pruebas. La notación de los parámetros elegidos y sus niveles se resumen en la tabla 2. Los en- 
sayos se realizaron de acuerdo con el plan definido por la tabla 3.

Tabla 2. Definición de los niveles de los parámetros

\begin{tabular}{lcc}
\hline & Nivel & Código \\
\hline \multirow{3}{*}{ Velocidad de corte } & 200 & 1 \\
& 100 & 2 \\
& 50 & 3 \\
\hline \multirow{3}{*}{ Velocidad de avance } & 0,05 & 1 \\
& 0,10 & 2 \\
& 0,15 & 3 \\
& 0,20 & 4 \\
\hline
\end{tabular}

Tabla 3. Plan de experimentos

\begin{tabular}{cll}
\hline Pruebas & $V_{c}$ & $V_{a}$ \\
\hline 1 & 1 & 1 \\
2 & 1 & 2 \\
3 & 1 & 3 \\
4 & 1 & 4 \\
5 & 2 & 1 \\
6 & 2 & 2 \\
7 & 2 & 3 \\
8 & 2 & 4 \\
9 & 3 & 1 \\
10 & 3 & 2 \\
11 & 3 & 3 \\
12 & 3 & 4 \\
\hline
\end{tabular}

Método de superficie de respuesta (MSR)

MSR es una herramienta utilizada para establecer la relación entre los factores controlables y las respuestas a fin de explorar el efecto de estos parámetros en las respuestas del proceso de mecanizado.

En muchas áreas de investigación interesa explicar una variable respuesta, $Y$, a través de $k$ variables explicativas, $x_{T}=\left[x_{1}, x_{2}, \ldots, x_{k}\right]$, mediante un modelo de la forma

$$
Y=f\left(x_{1}, x_{2}, \ldots, x_{k}\right)
$$

La función de respuesta se puede representar con una ecuación polinomial. El éxito en una investigación de una superficie de respuesta depende de que la respuesta se pueda ajustar a un polinomio de segundo grado.

El modelo de segundo orden es el siguiente

$$
Y=\beta_{0}+\sum_{i=1}^{k} \beta_{i} x_{i}+\sum_{i=1}^{k} \beta_{i i} x_{i}^{2}+\sum \sum_{i \prec j}^{k} \beta_{i j} x_{i} x_{j}
$$

Aquí los $\beta_{i}$ son los coeficientes de regresión para los términos de primer orden, los $\beta_{i i}$ son los coeficientes para los términos cuadráticos puros, los $\beta_{i j}$ son los coeficientes para los términos de producto cruz. Los términos cuadráticos puros y los de producto cruz son de segundo orden. El numero de términos en la ecuación está dado por

$p=(k+1)(k+2) / 2$

Para simplificar los cálculos, las variables independientes se codifican de la siguiente manera

$$
x_{i}^{\prime}=\left(x_{i}-m_{i}\right) / h_{i}
$$

$x_{j}^{\prime}=\left(x_{j}-m_{j}\right) / h_{j}$

donde $m_{i}$ es el rango medio de los valores $x_{i}, h_{i}=\left(x_{\text {imax }}+\right.$ $\left.x_{\text {imin }}\right) / 2$, y $m_{j}$ es el rango medio de los valores $x_{j}$ y $h_{j}=$ $\left(x_{\text {jmax }}+x_{\text {jmin }}\right) / 2$.

Para estimar los coeficientes se requieren $N \geq k+1$ valores de respuesta $(Y)$. El análisis de los datos de las corridas se presenta en una tabla de análisis de varianza. La tabla muestra las diferentes fuentes de variación que contribuyen a la variación total de los datos.

La variación total recibe el nombre de suma de cuadrados total SST y se calcula de la siguiente manera

$\mathrm{SST}=\sum_{u=1}^{N}\left(Y_{u}-\bar{Y}\right)^{2}$

donde $Y_{u}$ es el valor observado en el $u$-ésimo ensayo.

La suma de cuadrados se compone por la suma de cuadrados debido a la regresión y la suma de cuadrados residual. La fórmula de la suma de cuadrados debido a la regresión es

$\mathrm{SSR}=\sum_{u=1}^{N}\left(Y_{u}^{\prime}-\bar{Y}\right)^{2}$

La suma de cuadrados residual, se calcula de la siguiente forma

$\mathrm{SSE}=\sum_{u=1}^{N}\left(Y_{u}-\overline{Y_{u}}\right)^{2}$

A continuación la tabla 4 de varianza, presenta el número de términos del modelo ajustado. 
Tabla 4. ANOVA del modelo de regresión múltiple

\begin{tabular}{lccc}
\hline Fuente & $\begin{array}{c}\text { Grados de } \\
\text { libertad }\end{array}$ & $\begin{array}{c}\text { Suma de } \\
\text { cuadrados }\end{array}$ & $\begin{array}{c}\text { Media de } \\
\text { cuadrados }\end{array}$ \\
\hline Regresión & $p-1$ & SSR & SSR $/ p-1$ \\
Residuo & $N-p$ & SSE & SSE $/ N-p$ \\
Total & $N-1$ & SST & \\
\hline
\end{tabular}

La prueba de significancia de la ecuación de regresión ajustada tiene la siguiente hipótesis nula $\mathrm{H}_{0}$ : todas las $\beta_{\mathrm{s}}$ (excluyendo $\beta_{0}$ ) son cero contra la alternativa $\mathrm{H}_{\mathrm{A}}$. Al menos una de las $\beta_{\mathrm{s}}$ (excluyendo $\beta_{0}$ ) es diferente de cero. La prueba supone que el error se comporta normalmente y en ella se utiliza el estadístico de prueba $F$, el cual se calcula

$F=\frac{\operatorname{SSR} /(p-1)}{\operatorname{SSE} /(N-p)}$

Además de esta prueba se puede hacer un análisis del ajuste del modelo con la $R^{2}$, que es la proporción total de la variación de las $Y_{u}$ con respecto a la media que se puede explicar con la ecuación de regresión ajustada. Ésta se calcula de la siguiente manera

$R^{2}=\frac{\text { SSR }}{\text { SST }}$

En esta investigación, se han desarrollado modelos matemáticos de segundo orden basados en el método MSR para estudiar la influencia de los dos parámetros del proceso, a saber, la velocidad de corte $\left(V_{c}\right)$ y avance $\left(V_{a}\right)$, y las cuatro respuestas de rugosidad $\left(R_{t^{\prime}} R_{q^{\prime}}, R_{a^{\prime}}\right.$ y $\left.R_{p}\right)$. Las ecuaciones no lineales de la superficie de respuesta de $R_{t \prime} R_{q^{\prime}} R_{a^{\prime}} R_{p}$ son

$$
R_{i=t, q, a, p}=a_{0}+a_{1} V_{c}+a_{2} V_{a}+a_{3} V_{c} \cdot V_{a}+a_{4} V_{c}^{2}+a_{5} V_{a}^{2}
$$

con $a_{0}, a_{1}, a_{2}, \ldots, a_{5}$, como los coeficientes del modelo matemático a determinar.

Los valores de los coeficientes de regresión no lineal de la ecuación cuadrática (11) se determina a partir de

$B=\left(X^{t} X\right)^{-1} X^{t} Y$

donde

$B$ = la matriz de estimación de los parámetros de la ecuación cuadrática
$X=$ la matriz de cálculo que incluye términos lineales, cuadráticos y las interacciones

$X^{t}=$ la transposición de $X$ e

$Y=$ la matriz respuesta.

\section{Resultados}

Modelos matemáticos de los parámetros de rugosidad

Los modelos matemáticos de predicción para los parámetros de rugosidad de los materiales compuestos PEEK, PEEK FC 30 y PEEK GF 30 son los siguientes

$$
\begin{aligned}
& R_{t \text { (PEEK) }}=7.5833+0.0237 V_{c}+7.6474 V_{a} \\
& +0.3498 V_{c} \cdot V_{a}-0.0740 V_{c}^{2}+3.2155 V_{a}^{2} \\
& R_{q(\text { PEEK })}=2.1043-0.0451 V_{c}+2.3303 V_{a} \\
& +0.0016 V_{c} \cdot V_{a}-0.1151 V_{c}^{2}+0.7352 V_{a}^{2} \\
& R_{a(\text { PEEK })}=1.9621+0.0838 V_{c}+1.9166 V_{a} \\
& +0.1762 V_{c} \cdot V_{a}-0.3112 V_{c}^{2}+0.4913 V_{a}^{2} \\
& R_{p(\text { PEEK })}=4.7089-0.115 V_{c}+5.0764 V_{a} \\
& +0.2193 V_{c} \cdot V_{a}-0.0384 V_{c}^{2}+1.8937 V_{a}^{2} \\
& R_{t(\text { PEEK CF } 30)}=7.9772-0.575 V_{c}+5.5582 V_{a} \\
& -0.1782 V_{c} \cdot V_{a}+0.4152 V_{c}^{2}+2.6099 V_{a}^{2} \\
& R_{q(\text { PEEK CF } 30)}=1.5541-0.0850 V_{c}+1.4974 V_{a} \\
& -0.0236 V_{c} \cdot V_{a}+0.0909 V_{c}^{2}+0.6075 V_{a}^{2} \\
& R_{a(\text { PEEK СF30 })}=1.2801-0.0725 V_{c}+1.2606 V_{a} \\
& -0.0307 V_{c} \cdot V_{a}+0.0666 V_{c}^{2}+0.5100 V_{a}^{2} \\
& R_{p(\text { РЕEК СР30 })}=4.4227-0.3162 V_{c}+3.9606 V_{a} \\
& -0.2732 V_{c} \cdot V_{a}+0.2718 V_{c}^{2}+1.5824 V_{a}^{2} \\
& R_{t(\text { PEEK GF30 })}=15.955-0.865 V_{c}+6.5922 V_{a} \\
& -0.3674 V_{c} \cdot V_{a}-0.4575 V_{c}^{2}+2.7674 V_{a}^{2} \\
& R_{q(\text { PEEK GF30 })}=2.4330-0.1575 V_{c}+1.5111 V_{a} \\
& +0.0053 V_{c} \cdot V_{a}+0.0534 V_{c}^{2}+0.3619 V_{a}^{2} \\
& R_{a(\text { PEEK GF 30) }}=2.0169-0.1200 V_{c}+1.3027 V_{a} \\
& +0.0013 V_{c} \cdot V_{a}+0.0675 V_{c}^{2}+0.2306 V_{a}^{2} \\
& R_{p(P E E K ~ G F 30)}=8.2126-0.355 V_{c}+5.1033 V_{a} \\
& +0.0834 V_{c} \cdot V_{a}-1.3434 V_{c}^{2}+2.1299 V_{a}^{2}
\end{aligned}
$$


Tabla 5. Comparación de las predicciones y los valores experimentales del material PEEK

\begin{tabular}{ccclcccccccc}
\hline & \multicolumn{1}{c}{$\mathrm{R}_{t}$} & & \multicolumn{3}{c}{$\mathrm{R}_{q}$} & & & \multicolumn{2}{c}{$\mathrm{R}_{a}$} \\
\hline Exper. & Pred. & Residuos & Exper. & Pred. & Residuos & Exper. & Pred. & Residuos & Exper. & Pred. & Residuos \\
\hline 3.02 & 2.8993 & 0.1207 & 0.4 & 0.3473 & 0.0526 & 0.32 & 0.1331 & 0.1869 & 1.18 & 1.1534 & 0.0266 \\
5.64 & 5.3728 & 0.2672 & 1.24 & 1.2485 & -0.0085 & 1.02 & 1.0917 & -0.0717 & 3.44 & 3.0007 & 0.4393 \\
9.82 & 10.7038 & -0.8838 & 2.83 & 2.803 & 0.0270 & 2.48 & 2.4867 & -0.0067 & 5.54 & 6.5309 & -0.9909 \\
19.39 & 18.894 & 0.496 & 4.94 & 5.0112 & -0.0712 & 4.21 & 4.3186 & -0.1086 & 12.27 & 11.745 & 0.525 \\
3.38 & 3.2683 & 0.1117 & 0.55 & 0.5119 & 0.038 & 0.45 & 0.533 & -0.083 & 1.62 & 1.6334 & -0.0134 \\
4.47 & 5.4308 & -0.9608 & 0.92 & 1.4117 & -0.4917 & 0.72 & 1.3349 & -0.6149 & 2.76 & 3.2857 & -0.5257 \\
12 & 10.4509 & 1.5491 & 3.33 & 2.9648 & 0.3652 & 2.82 & 2.5734 & 0.2466 & 7.81 & 6.6209 & 1.1891 \\
17.63 & 18.33 & -0.7 & 5.26 & 5.1716 & 0.0884 & 4.7 & 4.2487 & 0.4513 & 10.99 & 11.64 & -0.65 \\
3.45 & 3.5516 & -0.1016 & 0.53 & 0.4407 & 0.0892 & 0.44 & 0.3179 & 0.1221 & 1.75 & 1.8221 & -0.0721 \\
5.86 & 5.5586 & 0.3014 & 1.3 & 1.3398 & -0.0398 & 1.05 & 1.0416 & 0.0084 & 3.64 & 3.377 & 0.2630 \\
10.15 & 10.4231 & -0.2731 & 3.04 & 2.8922 & 0.1478 & 2.64 & 2.2018 & 0.4382 & 6.24 & 6.6147 & -0.3747 \\
18.22 & 18.1467 & 0.0733 & 4.901 & 5.0983 & -0.1972 & 3.23 & 3.7988 & -0.5688 & 11.72 & 11.5362 & 0.1838 \\
\hline
\end{tabular}

Tabla 6. Comparación de las predicciones y los valores experimentales del material PEEK CF30

\begin{tabular}{|c|c|c|c|c|c|c|c|c|c|c|c|}
\hline \multicolumn{3}{|c|}{$\mathrm{R}_{t}$} & \multicolumn{3}{|c|}{$\mathrm{R}_{q}$} & \multicolumn{3}{|c|}{$\mathrm{R}_{a}$} & \multicolumn{3}{|c|}{$\mathrm{R}_{p}$} \\
\hline Exper. & Pred. & Residuos & Exper. & Pred. & Residuos & Exper. & Pred. & Residuos & Exper. & Pred. & Residuos \\
\hline 5.02 & 5.0475 & -0.0275 & 0.7 & 0.6937 & 0.0063 & 0.55 & 0.5543 & -0.0042 & 2.22 & 2.2734 & -0.0534 \\
\hline 5.93 & 6.3143 & -0.3843 & 1.13 & 1.1363 & -0.0063 & 0.92 & 0.9209 & -0.0009 & 3.15 & 3.3251 & -0.1751 \\
\hline 10.5 & 9.9007 & 0.5993 & 2.13 & 2.1187 & 0.0113 & 1.77 & 1.7408 & 0.0292 & 6.19 & 5.7832 & 0.4068 \\
\hline 15.62 & 15.8075 & -0.1875 & 3.63 & 3.6413 & -0.0113 & 2.99 & 3.0141 & -0.024 & 9.47 & 9.6483 & -0.1783 \\
\hline 5.26 & 5.2073 & 0.0527 & 0.68 & 0.6947 & -0.0147 & 0.55 & 0.5508 & -0.0008 & 2.02 & 2.0891 & -0.0691 \\
\hline 7.02 & 6.6326 & 0.3874 & 1.19 & 1.1583 & 0.0317 & 0.96 & 0.9448 & 0.0152 & 3.80 & 3.3837 & 0.4163 \\
\hline 10.2 & 10.3774 & -0.1774 & 2.09 & 2.1617 & -0.0717 & 1.72 & 1.7919 & -0.0719 & 5.77 & 6.0846 & -0.3146 \\
\hline 16.18 & 16.4427 & -0.2627 & 3.76 & 3.7053 & 0.0547 & 3.15 & 3.0925 & 0.0574 & 10.16 & 10.1926 & -0.0326 \\
\hline 5.98 & 5.841 & 0.139 & 0.82 & 0.8165 & 0.0035 & 0.64 & 0.6379 & 0.0021 & 2.44 & 2.3594 & 0.0806 \\
\hline 6.85 & 7.3455 & -0.4955 & 1.28 & 1.2905 & -0.0105 & 1.04 & 1.0454 & -0.0054 & 3.66 & 3.7755 & -0.1155 \\
\hline 11.24 & 11.1695 & 0.0705 & 2.35 & 2.3045 & 0.0455 & 1.94 & 1.9062 & 0.0337 & 6.38 & 6.5978 & -0.2178 \\
\hline 17.6 & 17.314 & 0.286 & 3.82 & 3.8585 & -0.0385 & 3.19 & 3.2205 & -0.0304 & 11.08 & 10.8272 & 0.2528 \\
\hline
\end{tabular}

Tabla 7. Comparación de las predicciones y los valores experimentales del material PEEK GF30

\begin{tabular}{|c|c|c|c|c|c|c|c|c|c|c|c|}
\hline \multicolumn{3}{|c|}{$\mathrm{R}_{t}$} & \multicolumn{3}{|c|}{$\mathrm{R}_{q}$} & \multicolumn{3}{|c|}{$\mathrm{R}_{a}$} & \multicolumn{3}{|c|}{$\mathrm{R}_{p}$} \\
\hline Exper. & Pred. & Residuos & Exper. & Pred. & Residuos & Exper. & Pred. & Residuos & Exper. & Pred. & Residuos \\
\hline 11.14 & 11.1751 & -0.0351 & 1.21 & 1.1744 & 0.0356 & 0.9 & 0.8911 & 0.0089 & 3.64 & 3.4574 & 0.1826 \\
\hline 13.07 & 12.8653 & 0.2047 & 1.76 & 1.8637 & -0.1037 & 1.51 & 1.5554 & -0.0454 & 5.83 & 5.0221 & 0.8079 \\
\hline 17.74 & 17.0147 & 0.7253 & 3 & 2.8746 & 0.1254 & 2.51 & 2.4246 & 0.0854 & 7.92 & 8.4796 & -0.5596 \\
\hline 22.73 & 23.6249 & -0.8949 & 4.15 & 4.2072 & -0.0572 & 3.45 & 3.4989 & -0.0489 & 13.4 & 13.8309 & -0.4309 \\
\hline 12.17 & 12.2453 & -0.0753 & 1.33 & 1.344 & -0.014 & 0.98 & 0.9928 & -0.0128 & 4.32 & 5.2361 & -0.9161 \\
\hline 13.31 & 14.262 & -0.952 & 2 & 2.0286 & -0.0286 & 1.62 & 1.656 & -0.036 & 6.27 & 6.7267 & -0.4567 \\
\hline 17.78 & 18.738 & -0.958 & 3.06 & 3.0347 & 0.0253 & 2.57 & 2.524 & 0.046 & 8.96 & 10.11 & -1.15 \\
\hline 27.66 & 25.6747 & 1.9853 & 4.38 & 4.3627 & 0.0173 & 3.6 & 3.5972 & 0.0028 & 17.91 & 15.3872 & 2.5228 \\
\hline 12.13 & 12.1704 & -0.0404 & 1.54 & 1.5 & 0.04 & 1.19 & 1.1336 & 0.0564 & 4.42 & 4.3343 & 0.0857 \\
\hline 15.55 & 14.3503 & 1.1997 & 2.13 & 2.1823 & -0.0523 & 1.72 & 1.7963 & -0.0763 & 7.38 & 5.7877 & 1.5923 \\
\hline 18.77 & 18.9897 & -0.2197 & 3.22 & 3.186 & 0.034 & 2.69 & 2.6637 & 0.0263 & 8.9 & 9.134 & -0.234 \\
\hline 25.15 & 26.0896 & -0.9396 & 4.49 & 4.5116 & -0.0216 & 3.73 & 3.7364 & -0.0064 & 12.93 & 14.3741 & -1.4441 \\
\hline
\end{tabular}

La comparación de las predicciones y los valores experimentales de $R_{t}, R_{q}, R_{a}$ y $R_{p}$ según el plan factorial completo para los materiales compuestos PEEK, PEEK CF30 y PEEK GF30 se muestra en las tablas 5, 6 y 7.

La figura 1 muestra la evolución de $R_{a}$ en función de los parámetros de corte para los dos tipos de herramientas utilizados en los ensayos. Como se puede ob- servar, es el PEEK GF30 reforzado con fibras de vidrio, el material con el que se obtiene un peor acabado superficial. El PEEK CF30 reforzado con fibras de carbono presenta valores ligeramente mayores en relación con el PEEK. La rugosidad aumenta significativamente al aumentar la velocidad de avance y disminuye ligeramente con la velocidad de corte. 


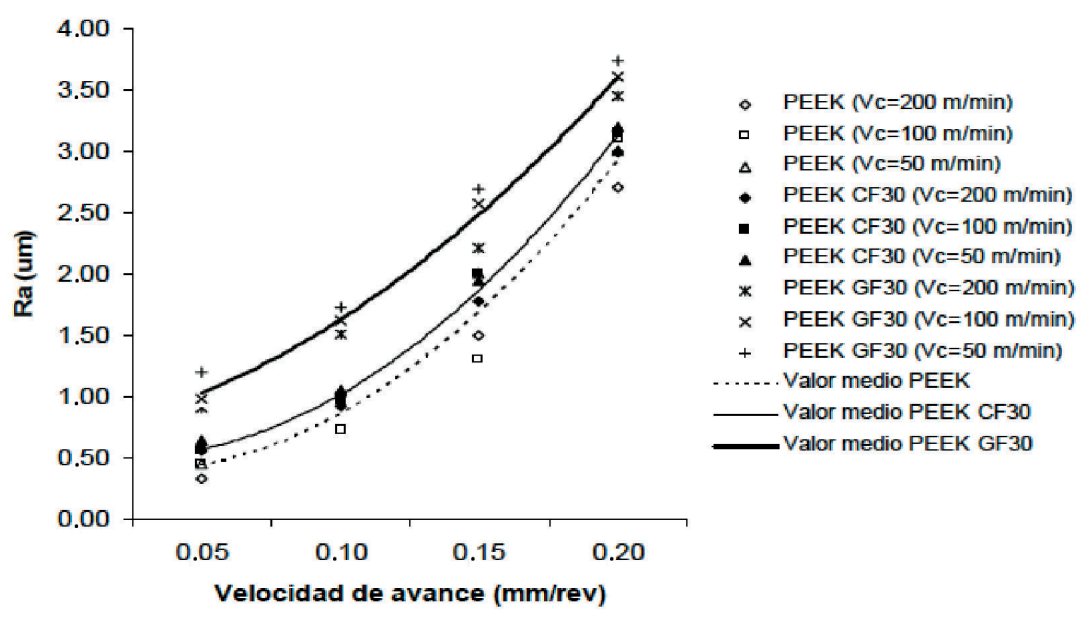

Figura 1. Evolución de $R_{a}$ en función de las velocidades de corte y avance
Figura 2. Evolución de $R_{t}$ en función de las velocidades de corte y avance

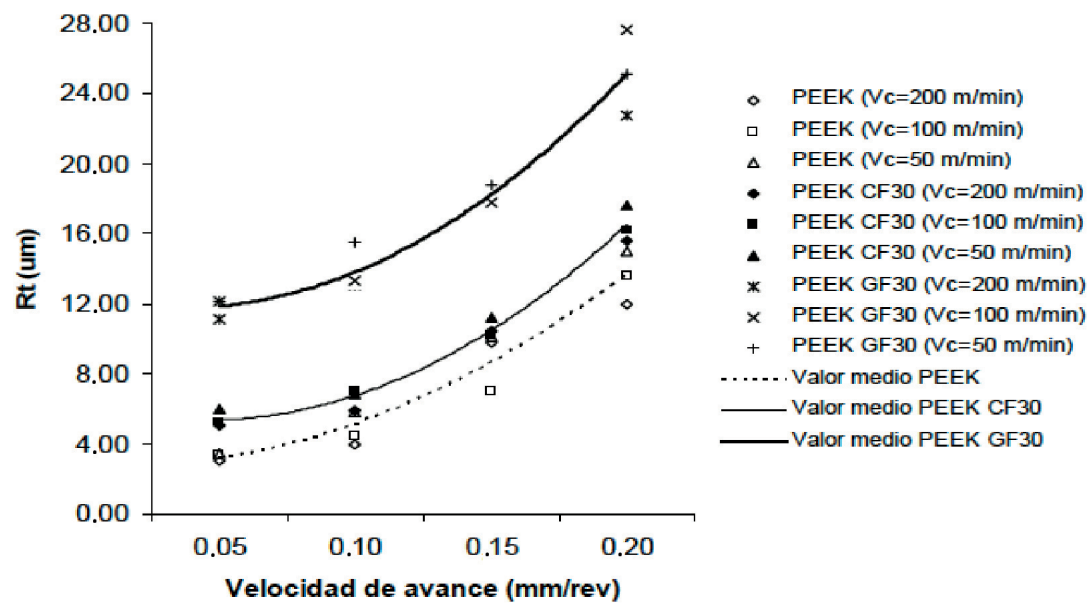

Trabajando con velocidades de avance bajas en los dos materiales se pueden conseguir calidades utilizadas en la construcción mecánica cuidada (IT7 e IT8) e incluso en la construcción de gran precisión (IT5 e IT6), especialmente para $V_{a}=0.05 \mathrm{~mm} / \mathrm{rev}$ en los tres materiales y también $V_{a}=0.10 \mathrm{~mm} / \mathrm{rev}$ en el caso del material no reforzado. Para velocidades de avance más elevadas $\left(V_{a}\right.$ $=0.15 \mathrm{~mm} / \mathrm{rev}$ y $V_{a}=0.20 \mathrm{~mm} / \mathrm{rev}$ ) se obtienen calidades aptas para construcción mecánica corriente (IT9 e IT10). En el caso del material no reforzado (PEEK) se obtienen casos de IT9 e IT10 sólo para $V_{a}=0.20 \mathrm{~mm} / \mathrm{rev}$. Por lo demás, el efecto de la velocidad de corte se puede considerar irrelevante.

\section{Verificación de los modelos matemáticos}

El análisis de varianza (ANOVA), tal como se explica por Montgomery, se utilizó para comprobar la adecuación de los modelos desarrollados para un determinado intervalo de confianza (Montgomery, 2003) y (Myers y 
Montgomery, 1995). El cuadro consta de ANOVA, suma de los cuadrados y los grados de libertad.

La adecuación de los modelos matemáticos desarrollados se presenta en el tabla 8. La calidad del ajuste de los modelos también fue probado por los coeficientes de determinación $\left(R^{2}\right)$ y $\left(R_{\text {Adj }}{ }\right)$. Los valores de $R^{2}$ y $R^{2}{ }_{\text {Adj }}$ de los modelos matemáticos no lineales (tabla 9) indican la correlación entre las variables independientes y las variables dependientes; además es necesario que los residuos deban ser distribuidos normalmente para que el análisis de regresión sea válido. Como ejemplo, las figuras 3, 4 y 5 presentan la probabilidad normal de $R_{a}$ y $R_{q}$ para los tres diferentes compuestos. Las figuras indican que los residuos siguen una línea recta, que denota una distribución normal. Resultados similares se obtienen para los casos de $R_{t}$ y $R_{p}$.

Tabla 8. Resultados de ANOVA de los modelos no lineales de los materiales compuestos

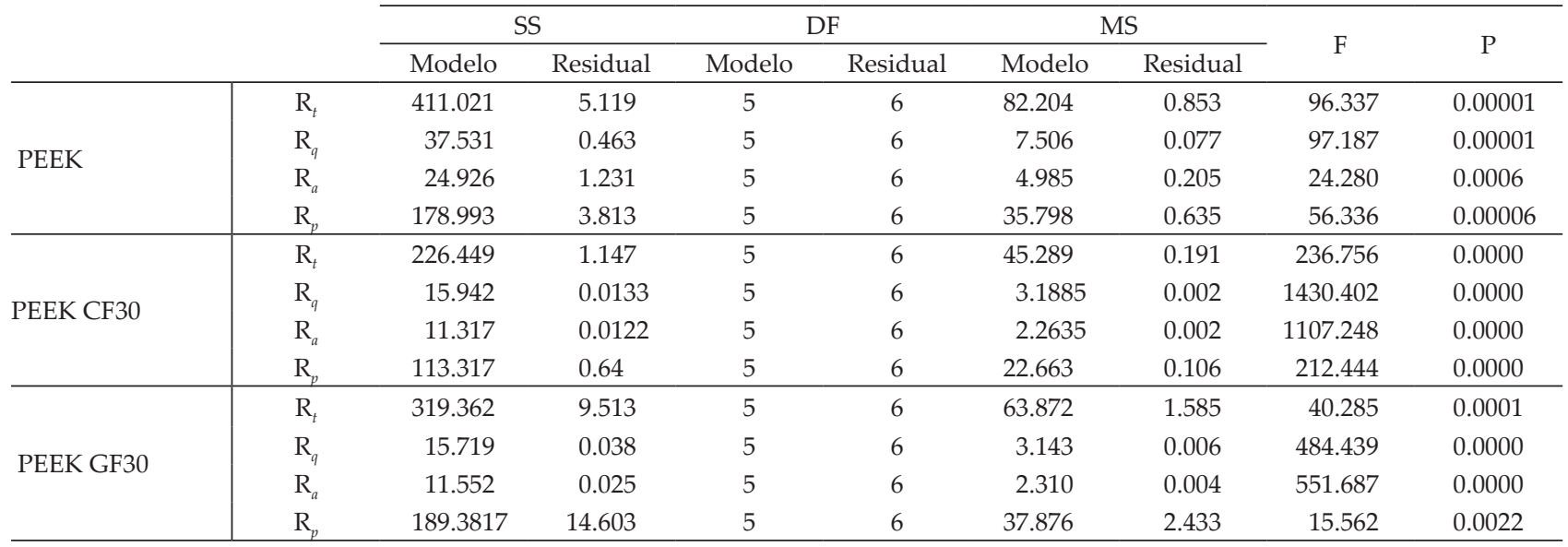

Tabla 9. Valores de $R^{2}$ y $R^{2}$ ajustada de los modelos no lineales de los materiales compuestos

\begin{tabular}{|c|c|c|c|c|c|c|}
\hline \multirow{3}{*}{ Respuesta } & \multicolumn{6}{|c|}{ Materiales } \\
\hline & \multicolumn{2}{|c|}{ PEEK } & \multicolumn{2}{|c|}{ PEEK CF30 } & \multicolumn{2}{|c|}{ PEEK GF30 } \\
\hline & $\mathrm{R}^{2}$ & $\mathrm{R}_{\mathrm{Adj}}^{2}$ & $\mathrm{R}^{2}$ & $\mathrm{R}_{\mathrm{Adj}}^{2}$ & $\mathrm{R}^{2}$ & $\mathrm{R}_{\mathrm{Adj}}^{2}$ \\
\hline $\mathrm{R}_{t}$ & 0.9878 & 0.9774 & 0.995 & 0.990 & 0.971 & 0.947 \\
\hline $\mathrm{R}_{q}$ & 0.9878 & 0.9776 & 0.9991 & 0.9984 & 0.9975 & 0.9954 \\
\hline $\mathrm{R}_{a}$ & 0.9529 & 0.9136 & 0.9989 & 0.9980 & 0.9978 & 0.9960 \\
\hline $\mathrm{R}_{p}$ & 0.979 & 0.962 & 0.994 & 0.989 & 0.928 & 0.868 \\
\hline
\end{tabular}

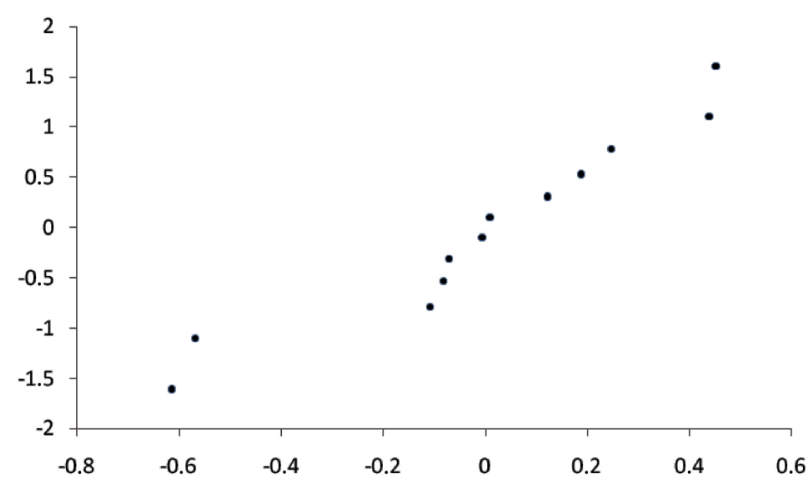

a)

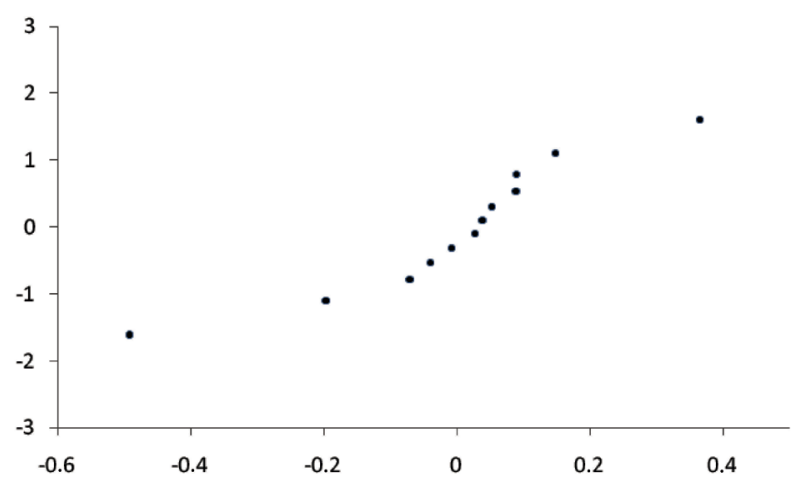

b)

Figura 3. Prueba de normalidad para los errores del modelo desarrollado por el PEEK: a) $R_{a}$; b) $R_{q}$ 


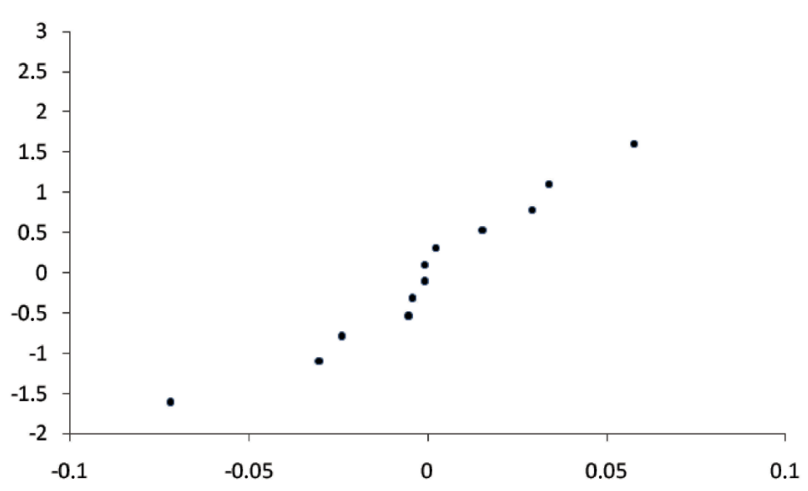

a)

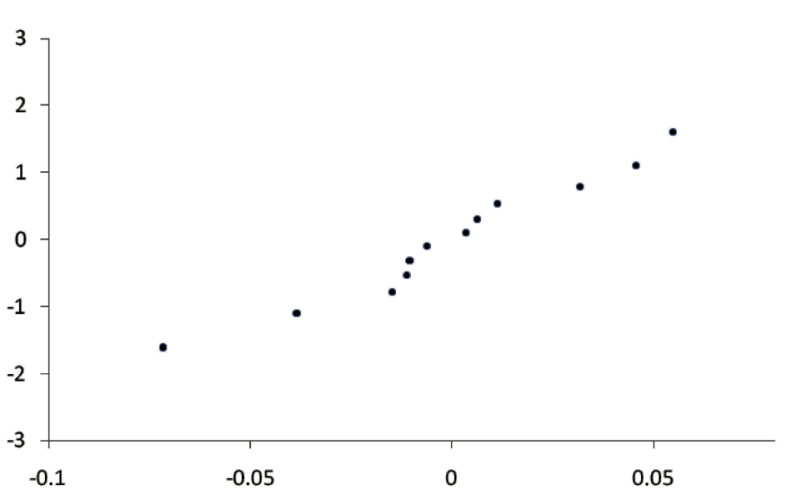

b)

Figura 4. Prueba de normalidad para los errores del modelo desarrollado por el PEEK CF30: a) $R_{a}$; b) $R_{q}$
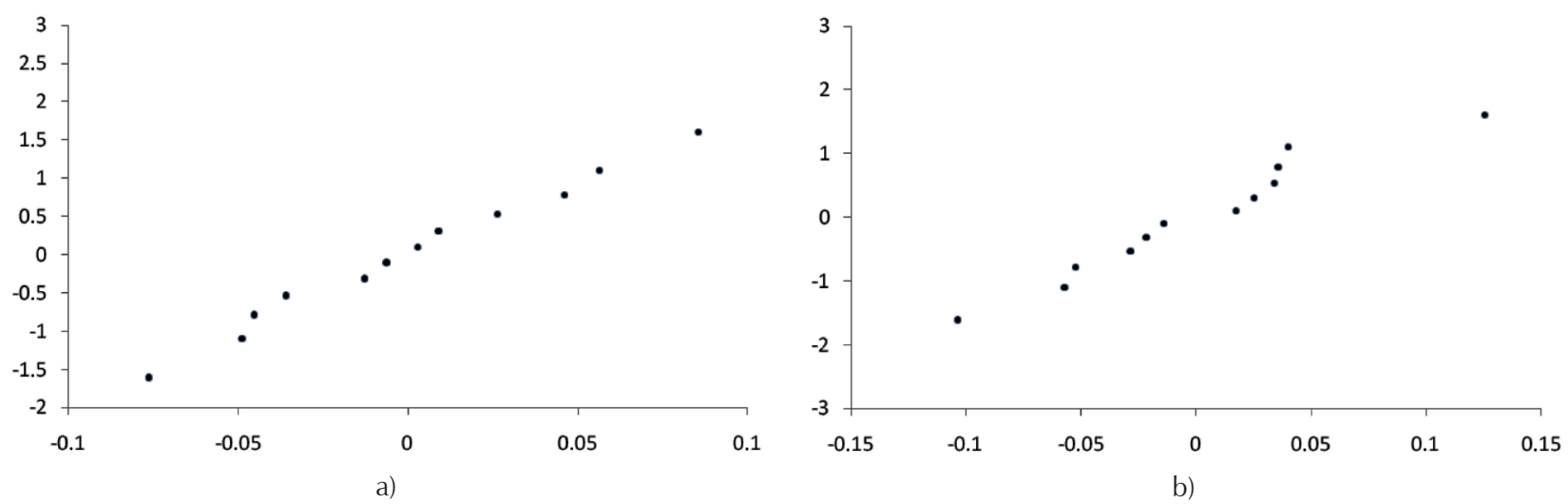

Figura 5. Prueba de normalidad para los errores del modelo desarrollado por el PEEK GF30: a) $R_{a}$; b) $R_{q}$

\section{Conclusiones}

Con el fin de analizar los efectos de los parámetros del proceso (velocidad de corte y velocidad de avance) en las respuestas $\left(R_{t}, R_{q^{\prime}} R_{a^{\prime}} R_{p}\right)$, los experimentos se planificaron según el método de diseño factorial completo.

En lo que respecta a la influencia de las condiciones de corte (parámetros funcionales de corte), se ha demostrado que la variación de la velocidad de avance, dentro de los valores seleccionados, afecta más a los resultados (fuerzas de corte, acabado superficial) que la variación de la velocidad de corte. A medida que aumenta la velocidad de avance, aumentan las fuerzas de corte y la rugosidad de las superficies mecanizadas, obteniéndose, por tanto, resultados peores.

Se ha verificado el efecto de las fibras de refuerzo en los dos grupos de materiales. La presencia de las fibras se traduce en mayores valores de las fuerzas de corte (fuerza de mecanizado, potencia de corte y fuerza específica de corte) y de la rugosidad $\left(R_{a} \mathrm{y} R_{t}\right)$. Este hecho es particularmente importante, ya que el mecanizado re- sulta más difícil, requiere mayor consumo de energía y el acabado es generalmente peor. Por tanto, los materiales reforzados presentan una peor maquinabilidad en comparación con el termoplástico sin refuerzo. En este caso, el PEEK GF30 reforzado con fibras de vidrio, es el material que evidencia una peor maquinabilidad.

Se han desarrollado modelos matemáticos no lineales de segundo orden para $R_{t}, R_{q^{\prime}} R_{a}$ y $R_{p}$ utilizando la metodología de superficie de respuesta. La calidad del ajuste de los modelos se ha probado por los coeficientes de determinación $R^{2}$ y $R_{\text {Adj' }}^{2}$ también se ha utilizado análisis de varianza para comprobar la suficiencia de los modelos. Los modelos de predicción han mostrado validez dentro del rango de parámetros de corte utilizados. Este trabajo de investigación propone el diseño y la implementación de modelos matemáticos tendientes a optimizar los procesos de mecanizado de elementos mecánicos. Las conclusiones a las que se llega tienen interés no sólo para futuras investigaciones que profundicen en ciertos aspectos o versen sobre cuestiones con otros enfoques complementarios, sino también para la 
industria, en la medida en que los resultados obtenidos plantean ciertas pautas a seguir desde el punto de vista práctico.

\section{Nomenclatura}

$R_{\mathrm{a}} \quad$ Rugosidad media $(\mu \mathrm{m})$

$R_{\mathrm{q}} \quad$ Media aritmética de las desviaciones del perfil $(\mu \mathrm{m})$

$R_{\mathrm{t}} \quad$ Distancia entre el punto más alto y el más bajo del registro $(\mu \mathrm{m})$

$R_{\mathrm{p}} \quad$ Distancia del punto más alto del perfil a la línea media $(\mu \mathrm{m})$

$R^{2} \quad$ Coeficiente de correlación

$R^{2}$ Adj $\quad$ Coeficiente de correlación ajustado

SS Suma de cuadrados

DF Grados de libertad

MS Varianza

F $\quad$ Test de Fischer

$P \quad$ Contribución

\section{Referencias}

Abouelatta O.B., Mádl J. Surface Roughness Prediction Based on Cutting Parameters and Tool Vibrations in Turning Operations. Journal of Materials Processing Technology, volumen 118, 2001: 269-277.

Bernardos P.G., Vosniakos C.G. Predicting Surface Roughness in Machining: a Review. International Journal of Machine Tools and Manufacture, volumen 43, 2003: 833-844.

Burris D.L. y Sawyer W.G. Tribological Behaviour of PEEK Components with Compositionally Graded PEEK/PTFE Surfaces. Wear, volumen 262, 2007: 220-224.

Cogswell F.N. Thermoplastic Aromatic Polymer Composites, Boston, Butterworh-Heinemann Ltd., 1992.

Davim J.P. y Mata F. Chemical Vapor Deposition (CVD) Diamond Coated Tools Performance in Machining of PEEK Composites. Materials and Design, volumen 29, 2008: 1568-1574.

Davim J.P. y Mata F. New Machinability Study of Glass Fiber Reinforced Plastics Using Polycrystalline Diamond and Cemented Carbide (K15) Tools. Materials \& Design, volumen 28, 2007: 1050-1054.

Davim J.P. y Mata F. Optimisation of Surface Roughness on Turning Fibre-Reinforced Plastics (FRPs) with Diamond Cutting Tools. International Journal of Advanced Manufacturing Technolo$g y$, volumen 26, 2005: 319-323.

Davim J.P. y Mata F. Physical Cutting Model of Polyetheretherketone Composites. Materials \& Design, volumen 27, 2006: 847-852.

Davim J.P. y Reis P. Dimensional Precision and Surface Roughness on Turning Tubes in Fiber Reinforced Plastics Based on the Design Experiments. International Journal of Materials and Product Technology, volumen 20, 2004: 268-279.
Davim J.P. y Reis P. Machinability Study on Composite (Polyetheretherketone Reinforced with 30\% Glass Fiber-PEEK GF 30) Uusing Polycrystalline Diamond (PCD) and Cemented Carbide (K20) Tools. International Journal of Advanced Manufacturing Technology, volumen 23, 2004: 412-418.

Davim J.P., Reis P., Lapa V., Conceicao C. Machinability Study on Polyetheretherketone (PEEK) Unreinforced and Reinforced (GF30) for Applications in Structural Components. Composite Structures, volumen 62, 2003: 67-73.

El-Sonbaty U.A., Khashaba T., Machaly T. Factors Affecting the Machinability of GFR/Epoxy Composites. Composite Structures, volumen 63, 2004: 329-338.

Eriksen E. Influence from Production Parameters on the Surface Roughness of a Machined Short Fibre Reinforced Thermoplastic. International Journal of Machine Tools and Manufacture, volumen 39, 1999: 1661-1618.

Harsha A.P., Tewari U.S. Two-Body and Three-Body Abrasive Wear Behavior Polyaryletherketone Composites. Polymer Testing, volumen 22, 2003: 403-408.

Kim B.C., Park D.C., Kim H.S., Lee D.G. Development of Composite Spherical Bearing. Composite Structures, volumen 75, 2006: 231-240.

Kukureka S.N., Hooke C.J., Rao M., Liao P., Chen Y.K. The Effect of Fibre Reinforcement on the Friction and Wear of Polyamide 66 Under Dry Rolling-Sliding Contact. Tribology International, volumen 32, 1999: 107-116.

Lee S.M., Park D.C., Kim B.C., Lee D.G. Strength of Functionally Gradient Composite Hemispherical Bearings. Composite Structures, volumen 74, 2006: 145-152.

Mallick V. Thermoplastic Composite Based Processing Technologies for High Performance Turbomachinery omponents, Composites Part A. Applied Science and Manufacturing (Incorporating Composites and Composites Manufacturing), volumen 32, 2001: 1167-1173.

Miravete A., Larrodé E., Castejón L., Clemente R., et al. Materiales compuestos (tomos I y II), editorial AM, 2000.

Montgomery D.C. Design and Analysis of Experiments, New York, John Wiley and Sons, 2003.

Myers R.H., Montgomery D.C. Response Surface Methodology: Process and Product Optimization using Designed Experiments, New York, John Wiley and Sons, 1995.

Palanikumar K. Cutting Parameters Optimization for Surface Roughness in Machining of GFRP Composites Using Taguchi's Method. Journal of Reinforced Plastics and Composites, volumen 25, 2006: 1739-1751.

Palanikumar K., Karunamoorthy L., Karthikeyan R. Optimizing the Machining Parameters for Minimum Surface Roughness in Turning of GFRP Composites Using Design of Experiments. Journal of Materials Science and Technology, volumen 20, 2004: 373-378, 2004.

Palanikumar K., Karunamoorthy L., Karthikeyan R. Parametric Optimization to Minimise the Surface Roughness on the Ma- 
chining of GFRP Composites. Journal of Materials Science and Tecnholology, volumen 22, 2006: 66-72.

Park D.C., Lee S.M., Kim B.C., Kim H.S., Lee D.G. Development of Heavy Duty Hybrid Carbon-Phenolic Hemispherical Bearings. Composite Structures, volumen 73, 2006: 88-98.

Petropoulos G., Davim JP., Mata F., Pandazaras C. New Considerations of Evaluating the Anisotropy of Machined Surfaces. Journal of the Balkan Tribological Association, volumen 12, 2006: 1-6.

Sinmazcelik T., Yilmaz T. Thermal Aging Effects on Mechanical and Tribological Performance of PEEK and Short Fiber Reinforced PEEK Composites. Materials and Design, volumen 28, 2007: 641-648.

Taguchi G. Introduction to Quality Engineering, Tokyo, Asian Productivity Organization, 1990.

Varatharajan R., Malhotra S.K., Vijayaraghavan L., Krishnamurthy R. Mechanical and Machining Characteristics of GF/PP and GF/Polyester Composites. Materials Science and Engineering $B$, volumen 132, 2006: 134-137.

Verrey J., Wakeman M.D., Michaud V., Manson J.A.E. Manufacturing Cost Comparison of Thermoplastic and Thermoset RTM for an Automotive Floor Pan. Composites Part A: Applied Science and Manufacturing, volumen 37, 2006: 9-22.
Yang W.H. y Tarng Y.S. Design Optimization of Cutting Parameters for Turning Operations Based on the Taguchi Method. Journal of Materials Processing Technology, volumen 84, 1998: 122-129.

\section{Este artículo se cita:}

\section{Citación estilo Chicago}

Mata-Cabrera, Francisco, Issam Hanafi, Abdellatif Khamlichi, Abdallah Jabbouri, Mohamed Bezzazi. Predicción de los parámetros de rugosidad en elementos de máquina mecanizados en compuestos de Peek usando metodología de superficie de respuesta. Ingeniería Investigación y Tecnología, XIV, 04 (2013): 463-474.

\section{Citación estilo ISO 690}

Mata-Cabrera F., Hanafi I., Khamlichi A., Jabbouri A., Bezzazi M. Predicción de los parámetros de rugosidad en elementos de máquina mecanizados en compuestos de Peek usando metodología de superficie de respuesta. Ingeniería Investigación y Tecnología, volumen XIV (número 4), octubre-diciembre 2013: 463-474.

\section{Semblanza de los autores}

Francisco Mata-Cabrera. Es ingeniero técnico industrial (mecánico), ingeniero técnico de minas (explotación de minas), ingeniero mecánico e ingeniero industrial. Asimismo tiene maestría en prevención de riesgos laborales, master en dirección de recursos humanos, maestría en evaluación de impacto ambiental y doctor en ingeniería mecanica por la Universidade de Aveiro (Portugal). Actualmente es director de la Escuela de Ingeniería Minera e Industrial de Almadén, España. Sus áreas de interés son modelización de mecanizado de materiales avanzados de matriz polimérica, análisis de sistemas vibrantes, simulación y diseño de componentes para automoción.

Issam Hanafi. Es doctor de estado marroquí en mecánica por la Facultad de Ciencias, Tetuán, Marruecos (2011). Realizó estudios de ingeniería civil en la Facultad de Ciencias y Técnicas de Tanger, Marruecos, recibiendo su título en 2005. También, obtuvo su diploma de estudios superiores en mecánica en 2007 por parte de la Facultad de Ciencias de Tétouan, Marruecos. Sus áreas de interés son mecánica estructural, mecánica de materiales e ingeniería civil.

Abdellatif Khamlichi. Es doctor de estado marroquí en mecánica por la Facultad de Ciencias, Tetuán, Marruecos (2004) y doctorado francés en mecánica por la Escuela Centrale de Lyon, Francia (1992). Actualmente es profesor-investigador en la Facultad de Ciencias, Tetuán, Marruecos. Sus áreas de interés son mecánica estructural, mecánica de materiales, ingeniería civil, mecatrónica, erosión interna en los suelos.

Abdallah Jabbouri. Obtuvo el doctorado del estado por la Universidad de Ciencias y Tecnicas de Languedoc (USTL)-Montpellier, Francia. Actualmente es profesor-investigador en la Facultad de Ciencias y Técnicas, Tanger, Maruecos. Sus áreas de interés son mecánica estructural, mecánica de materiales, ingeniería civil, mecatrónica, erosión interna en los suelos.

Mohamed Bezzazi. Es doctorado francés en ingénieria civil por la Universidad de Lille, Francia. Actualmente es profesor-investigador en la Facultad de Ceincias y Tecnicas, Tanger, Maruecos. Sus áreas de interés son mecánica estructural, mecánica de materiales, ingeniería civil, mecatrónica y erosión interna en los suelos. 